\title{
High Resolution Channel Quantization Rules for Multiuser Spatial Multiplexing Systems
}

\author{
Behrouz Khoshnevis and Wei Yu \\ Department of Electrical and Computer Engineering \\ University of Toronto, Toronto, Ontario, Canada \\ Email: \{bkhoshnevis, weiyu\}@comm.utoronto.ca
}

\begin{abstract}
This paper addresses the optimal channel quantization codebook design for limited feedback multiple-antenna multiuser channels. The base station is equipped with $M$ antennas and serves $M$ single-antenna users, which share a total feedback rate $B$. We assume real space channels for convenience; the extension of the analysis to complex space is straightforward. The codebook optimization problem is cast in form of minimizing the average downlink transmission power subject to the users' outage probability constraints. This paper adopts a product codebook structure for channel quantization comprising a uniform (in $\mathrm{dB}$ ) channel magnitude quantization codebook and a spatially uniform channel direction quantization codebook. We first formulate a robust power control problem that minimizes the sum power subject to the worst-case SINR constraints over the channel quantization regions. By using an upper bound solution to this problem, we then optimize the quantization codebooks given the target outage probabilities and the target SINR's. In the asymptotic regime of $B \rightarrow \infty$, the optimal number of channel direction quantization bits is shown to be $M-1$ times the number of channel magnitude quantization bits. It is further shown that the users with higher requested QoS (lower target outage probabilities) and higher requested downlink rates (higher target SINR's) should receive larger shares of the feedback rate. The paper also shows that, for the target parameters to be feasible, the total feedback bandwidth should scale logarithmically with $\bar{\gamma}$, the geometric mean of the target SINR values, and $1 / \bar{q}$, the geometric mean of the inverse target outage probabilities. Moreover, the minimum required feedback rate increases if the users' target parameters deviate from the average parameters $\bar{\gamma}$ and $\bar{q}$. Finally, we show that, as $B$ increases, the multiuser system performance approaches the performance of the perfect channel state information system as $1 / \bar{q} \cdot 2^{-\frac{B}{M^{2}}}$.
\end{abstract}

\section{INTRODUCTION}

The availability of channel state information (CSI) at the transmitter is critical for the operation of the multiuser spatial multiplexing systems. The base station needs this information to distinguish the users spatially and perform power control. In practice, this information is provided either by training the base station on the reverse links in TDD systems or by quantizing the user channels and sending back the quantized information in FDD systems. The design and optimization of the channel quantization codebooks, therefore, is essential in the implementation of the FDD systems.

The multiple-antenna multiuser system with imperfect CSI at the transmitter is a well investigated topic in the literature [1]-[5]. In order to preserve the multiplexing gain of the sum rate, the author of [2] shows that the feedback rate should scale linearly with the SNR (in dB). The authors of
[3] show that one needs the channel magnitude information in addition to the quantized channel direction information in order to realize the multiuser diversity gain of the sum rate in a large network of users. The magnitude information, however, is assumed to be perfect in [3]. The joint scheduling and beamforming problem with a total feedback rate constraint across users is studied by [4]. Finally, the authors of [5] study the throughput optimization problem considering both training and CSI feedback overheads.

A majority of the literature in the area of multiuser feedback design considers the sum rate as the performance metric and, for tractability reasons, does not allow power adaptation over time and across the users. In practical systems, however, temporal and spatial power adaptation are essential as the users request instantaneous downlink data rates with specific QoS constraints. Therefore, the base station needs not only the channel direction information for spatial identification of the users, but also the channel magnitude information for power control and/or rate control. It is therefore necessary to study the joint direction and magnitude quantization codebook design and optimization.

To address this problem, we formulate the system design problem as minimization of the average sum power subject to the outage probability constraints at the users' side. Such formulation is appropriate for fixed-rate delay-sensitive type of traffic. This paper adopts a product codebook structure comprising a spatially uniform direction quantization codebook and a uniform (in $\mathrm{dB}$ ) channel magnitude quantization codebook; the asymptotic optimality of such uniform (in $\mathrm{dB}$ ) magnitude codebooks is shown in [6]. The product structure has several practical advantages [7] and it is shown to be a sufficient structure for effective interference management in the multiuser systems [8].

Assuming zero-forcing beamforming vectors, this paper starts by formulating the robust power control problem subject to the worst-case SINR constraints over the sector-type quantization regions imposed by the product quantization structure. Using an upper bound solution to this problem, we then study the optimization of the quantization codebooks for the given target SINR's and outage probabilities. The analysis is asymptotic in the total feedback rate. We show that

1) The optimal number of direction quantization bits is $M-1$ times the number of magnitude quantization bits, where $M$ is the number of base station antennas. 
2) The share of the $k$ th user from the total feedback bandwidth is controlled by $\log \gamma_{k}$ and $\log 1 / q_{k}$, where $\gamma_{k}$ and $q_{k}$ are the user's target SINR and outage probability. As a general rule, a user with a higher QoS (lower target outage probability) and higher target rate (higher target SINR) needs a higher channel quantization resolution and therefore requires a larger share of the total feedback rate.

3) For the outage probability constraints to be feasible, the total feedback bandwidth should scale with $\log \bar{\gamma}$ and $\log 1 / \bar{q}$, where $\bar{\gamma}$ and $\bar{q}$ are the geometric means of the target SINR's and target outage probabilities. Moreover, the minimum required feedback rate increases if the users' target parameters deviate from the average parameters $\bar{\gamma}$ and $\bar{q}$, i.e. there is a feedback rate penalty for serving users with different target parameters. The higher the deviation, the higher is the penalty.

4) As the total feedback rate $B$ increases, the performance of the limited CSI system approaches the performance of the perfect CSI system as $\frac{1}{\bar{q}} \cdot 2^{-\frac{B}{M^{2}}}$.

The proofs of the theorems in this paper are omitted due to the space limits. Also, all the computations in the paper are for the real space. Extension of the results to the complex space is straightforward.

\section{Perfect CSI System: Outage is IneVitable}

This section provides a quick review of spatial multiplexing system and shows that outage is inevitable even with perfect CSI at the base station. The discussions are adopted from [8].

Consider a multiuser downlink channel with $M$ antennas at the base station and $M$ users each with a single antenna. Let $\mathbf{h}_{k} \in \mathbb{R}^{M}, \mathbf{v}_{k} \in \mathbb{R}^{M}, P_{k}$, and $\gamma_{k}$ denote respectively, the user channel, the beamforming vector, the allocated power, and the target SINR for the $k$ th user, $1 \leq k \leq M$. The minimization of the transmission sum power subject the user SINR constraints is formulated as follows:

$$
\begin{aligned}
& \min _{P_{k}, \mathbf{v}_{k}} \sum_{k=1}^{M} P_{k} \\
& \text { s.t. } \frac{P_{k}\left|\mathbf{h}_{k}^{T} \mathbf{v}_{k}\right|^{2}}{\sum_{l \neq k} P_{l}\left|\mathbf{h}_{k}^{T} \mathbf{v}_{l}\right|^{2}+1} \geq \gamma_{k}, \quad k=1,2, \cdots, M
\end{aligned}
$$

where the receiver noise power is assumed to be 1 for all users.

A suboptimal solution for problem (1) is to use zero-forcing (ZF) beamforming vectors $\mathbf{v}_{k}$ to eliminate the interference and find the power values $P_{k}$ that satisfy the constraints with equality. This solution is asymptotically optimal in the high SNR regime. An important matter to consider with this solution is that the transmission powers need to be very high when the users' channels are closely aligned, as the $\mathrm{ZF}$ beamforming vectors would be almost perpendicular to the corresponding channels in such cases. Therefore, it is not possible to always satisfy the SINR constraints with a bounded average power and, as a result, a certain degree of outage should be tolerated by the users.

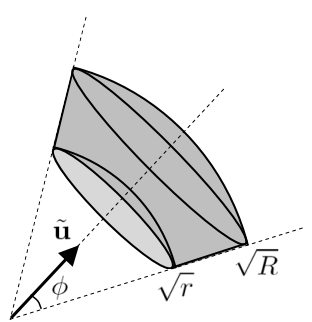

Fig. 1. Sector-type quantization region $S(R, r, \tilde{\mathbf{u}}, \phi)$.

To see this rigorously, define $\theta_{k}=\angle\left(\mathbf{h}_{k}, \mathbf{H}_{-k}\right)$, where $0 \leq$ $\theta_{k} \leq \frac{\pi}{2}$, and $\mathbf{H}_{-k}=\operatorname{span}\left(\left\{\mathbf{h}_{l} \mid l \neq k\right\}\right)$. Assume that the users' channels are i.i.d. with uniformly distributed directions and independent channel magnitudes (with arbitrary distributions). The average sum power of $\mathrm{ZF}$ method is given by

$$
P_{\mathrm{CSI}}=\mathbb{E}\left\{P_{\mathrm{ZF}, \text { sum }}\right\}=\sum_{k=1}^{M} \gamma_{k} \mathbb{E}\left\{1 /\left\|\mathbf{h}_{k}\right\|^{2}\right\} \mathbb{E}\left\{1 / \sin ^{2}\left(\theta_{k}\right)\right\} .
$$

As $\theta_{k}$ is uniformly distributed in $\left[0, \frac{\pi}{2}\right]$, the expectation of $1 / \sin ^{2}\left(\theta_{k}\right)$ becomes unbounded. To avoid unbounded transmit power, the users should therefore tolerate certain degrees of outage. A reasonable approach is to declare outage for user $k$, i.e. set $P_{k}=0$, when $0 \leq \theta_{k} \leq \theta_{k}^{\circ}$, where $\theta_{k}^{\circ} \ll 1$ is the smallest acceptable angle between $\mathbf{h}_{k}$ and $\mathbf{H}_{-k}$. With this assumption the average sum power is given by

$$
P_{\mathrm{CSI}}=\frac{2}{\pi} \sum_{k=1}^{M} \gamma_{k} \cot \left(\theta_{k}^{\circ}\right) \mathbb{E}\left\{1 /\left\|\mathbf{h}_{k}\right\|^{2}\right\} \approx \frac{2 \rho_{\mathrm{CSI}}}{\pi} \sum_{k=1}^{M} \frac{\gamma_{k}}{\theta_{k}^{\circ}},
$$

where

$$
\rho_{\mathrm{CSI}} \stackrel{\text { def }}{=} \mathbb{E}\left\{1 /\left\|\mathbf{h}_{k}\right\|^{2}\right\}
$$

for i.i.d. users. The corresponding outage probabilities are $p_{\text {out }, k}=2 \theta_{k}^{\circ} / \pi$ for $1 \leq k \leq M$.

\section{Robust Power Control With Sector-Type CHANNEL UNCERTAINTY REGIONS}

The optimization of the multiuser spatial multiplexing system with quantized channel information is a two-fold problem: 1) optimizing the power control and beamforming functions for fixed quantization codebooks; 2) optimization of the quantization codebooks. In this section we study the first problem; the codebook optimization will be discussed later.

For product quantization codebooks that are considered in this paper, the quantization (or channel uncertainty) regions are the sector-type regions shown in Fig. 1:

$S(R, r, \tilde{\mathbf{u}}, \phi)=\left\{\mathbf{h} \in \mathbb{R}^{M} \mid \sqrt{r} \leq\|\mathbf{h}\|<\sqrt{R}, \angle(\mathbf{h}, \tilde{\mathbf{u}})<\phi\right\}$,

where $\tilde{\mathbf{u}}$ is the quantized direction and $\phi$ is the uncertainty angle.

Define the uncertainty regions $S_{k}=S\left(R_{k}, r_{k}, \tilde{\mathbf{u}}_{k}, \phi_{k}\right)$ for users $1 \leq k \leq M$. We are interested in minimizing the sum 
power subject to the worst-case SINR constraints over these regions:

$$
\begin{aligned}
& \min _{P_{k}} \sum_{k=1}^{M} P_{k} \\
& \text { s.t. } \inf _{\mathbf{w} \in S_{k}} \frac{P_{k}\left|\mathbf{w}^{T} \mathbf{v}_{k}\right|^{2}}{\sum_{l \neq k} P_{l}\left|\mathbf{w}^{T} \mathbf{v}_{l}\right|^{2}+1} \geq \gamma_{k}, \quad k=1,2, \cdots, M
\end{aligned}
$$

The beamforming vectors $\mathbf{v}_{k}$ are assumed to be the zeroforcing vectors for the quantized directions $\tilde{\mathbf{u}}_{k}$ and the optimization is only over the power control functions.

This problem can be transformed to a SDP problem and therefore efficiently solved [9]. In order to obtain a closedform answer for the sum power, we resort to a suboptimal solution that leads to an upper bound on the sum power. This bound is used in the later sections for the optimization of the quantization codebooks. A byproduct of this upper bound solution is a sufficient feasibility condition for the original problem in (4).

Define $\theta_{k}=\angle\left(\tilde{\mathbf{u}}_{k}, \tilde{\mathbf{U}}_{-k}\right)$, which is a similar definition as in Section II, except that the exact channels $\mathbf{h}_{k}$ 's are replaced with the quantized directions $\tilde{\mathbf{u}}_{k}$ 's. First, we bound the SINR terms as follows:

$$
\begin{aligned}
\inf _{\mathbf{w} \in S_{k}} \frac{P_{k}\left|\mathbf{w}^{T} \mathbf{v}_{k}\right|^{2}}{\sum_{l \neq k} P_{l}\left|\mathbf{w}^{T} \mathbf{v}_{l}\right|^{2}+1} & \stackrel{(\text { a) }}{=} \inf _{\mathbf{w} \in S_{k}} \frac{P_{k} r_{k}\left|\hat{\mathbf{w}}^{T} \mathbf{v}_{k}\right|^{2}}{r_{k} \sum_{l \neq k} P_{l}\left|\hat{\mathbf{w}}^{T} \mathbf{v}_{l}\right|^{2}+1} \\
& \geq \frac{P_{k} r_{k} \inf _{\mathbf{w} \in S_{k}}\left|\hat{\mathbf{w}}^{T} \mathbf{v}_{k}\right|^{2}}{r_{k} \sum_{l \neq k} P_{l} \sup _{\mathbf{w} \in S_{k}}\left|\hat{\mathbf{w}}^{T} \mathbf{v}_{l}\right|^{2}+1} \\
& \stackrel{(\mathrm{b})}{=} \frac{P_{k} r_{k} \sin ^{2}\left(\theta_{k}-\phi_{k}\right)}{\left(\sum_{l=1}^{M} P_{l}\right) r_{k} \sin ^{2} \phi_{k}+1}
\end{aligned}
$$

where $\hat{\mathbf{w}}=\mathbf{w} /\|\mathbf{w}\|$ in (5). The equality (a) holds since the SINR term is monotonic in $\|\mathbf{w}\|$, i.e. the minimum occurs on the smaller hypersphere in Fig. 1. The equality (b) is a direct result of the definitions of $\theta_{k}, S_{k}$, and the fact that $\mathbf{v}_{k}$ 's are the zero-forcing directions for $\tilde{\mathbf{u}}_{k}$ 's.

Now, by setting the last term in (6) to be equal to $\gamma_{k}$, one achieves a set of linear equations in $P_{k}$ 's and by solving these equations we have the following upper bound for the sum power:

$$
P_{u b}=\frac{\sum_{k=1}^{M} \alpha_{k} / \beta_{k}}{1-\sum_{k=1}^{M} \alpha_{k}},
$$

where $\alpha_{k}=\left(1+\frac{\sin ^{2}\left(\theta_{k}-\phi_{k}\right)}{\gamma_{k} \sin ^{2} \phi_{k}}\right)^{-1}$ and $\beta_{k}=r_{k} \sin ^{2} \phi_{k}$.

The method used above for deriving the upper bound (7) is similarly used by [8] for spherical channel uncertainty regions instead of sector-type regions.

By guaranteeing $\sum_{k=1}^{M} \alpha_{k}<1$ in (7), one can prove the following sufficient feasibility condition for the original problem in (4).

Theorem 1: To ensure the feasibility of the problem in (4), it is sufficient to have

$$
\frac{\tan \phi_{k}}{\sin \theta_{k}}<\frac{1}{1+\sqrt{(M-1) \gamma_{k}}}
$$

For small values of uncertainty angles $\phi_{k}$, this is equivalent to

$$
\phi_{k}<\frac{\sin \theta_{k}}{1+\sqrt{(M-1) \gamma_{k}}} .
$$

Since we are interested in computing the expected value of the sum power, we use the following approximation for the sum-power upper bound so that the expectation operation can be conveniently applied:

Theorem 2: For $\phi_{k} \ll 1,1 \leq k \leq M$, we have

$$
P_{u b}=\sum_{k} e_{k}+\sum_{k} f_{k} \phi_{k}+\sum_{k} o\left(\phi_{k}\right)
$$

where

$$
e_{k}=\frac{\gamma_{k}}{r_{k}}\left(1+\zeta_{k}^{2}\right), \quad f_{k}=\frac{2 \gamma_{k}}{r_{k}}\left(\zeta_{k}+\zeta_{k}^{3}\right), \quad \zeta_{k}=\cot \theta_{k} .
$$

The assumption of $\phi_{k} \ll 1$ is justified in Section V.B.

In the following sections, we use the sufficient condition (9) and the sum-power upper bound approximation (10) to optimize the channel quantization codebook.

\section{Quantization Codebook Optimization: The GENERAL FORM}

In this section, we present the codebook optimization problem in its general form. To clarify the arguments, we start by some basic definitions.

By a quantization codebook $\mathcal{C}=\left\{S^{(1)}, S^{(2)}, \cdots, S^{(N)}\right\}$ of size $N$, we mean a partition of $\mathbb{R}^{M}$ into $N$ disjoint quantization regions $S^{(n)}, 1 \leq n \leq N$. For every quantization codebook $\mathcal{C}$, we also define a quantization function $\mathcal{S}(\mathbf{h}): \mathbb{R}^{M} \rightarrow \mathcal{C}$ that returns the quantization region that $\mathbf{h} \in \mathbb{R}^{M}$ belongs to.

Now for $M$ users $1 \leq k \leq M$, consider $M$ codebooks $\mathcal{C}_{k}$ of sizes $N_{k}$ and the corresponding quantization functions $\mathcal{S}_{k}\left(\mathbf{h}_{k}\right)$, where $\mathbf{h}_{k}$ is the $k$ th user's channel. Define the ordered $M$ tuples

$$
\begin{aligned}
& \mathbf{H}=\left[\mathbf{h}_{1}^{T}, \mathbf{h}_{2}^{T}, \cdots, \mathbf{h}_{M}^{T}\right] \in \mathbb{R}^{M^{2}} \\
& \mathcal{S}(\mathbf{H})=\left[\mathcal{S}_{1}\left(\mathbf{h}_{1}\right), \mathcal{S}_{2}\left(\mathbf{h}_{2}\right), \cdots, \mathcal{S}_{M}\left(\mathbf{h}_{M}\right)\right] \in \prod_{k=1}^{M} \mathcal{C}_{k} .
\end{aligned}
$$

For a given total number of quantization (feedback) bits $B$, target SINR values $\gamma_{k}$, and target outage probabilities $q_{k}$, the quantization codebook optimization problem is formulated as follows:

$$
\begin{array}{ll}
\min _{\substack{\mathcal{C}_{k}, N_{k}, P_{k}(\mathcal{S}(\mathbf{H})), \mathbf{v}_{k}(\mathcal{S}(\mathbf{H}))}} & \mathbb{E}_{\mathbf{H}}\left[\sum_{k=1}^{M} P_{k}(\mathcal{S}(\mathbf{H}))\right] \\
\text { s.t. } & \prod_{k=1}^{M} N_{k} \leq 2^{B}, \\
\operatorname{prob} & {\left[\frac{P_{k}(\mathcal{S}(\mathbf{H}))\left|\mathbf{h}_{k}^{T} \mathbf{v}_{k}(\mathcal{S}(\mathbf{H}))\right|^{2}}{\sum_{l \neq k} P_{l}(\mathcal{S}(\mathbf{H}))\left|\mathbf{h}_{k}^{T} \mathbf{v}_{l}(\mathcal{S}(\mathbf{H}))\right|^{2}+1}<\gamma_{k}\right]<q_{k},} \\
k=1,2, \cdots, M
\end{array}
$$


where the optimization is over the quantization codebooks $\mathcal{C}_{k}$, codebook sizes $N_{k}$, the power control functions $P_{k}(\mathcal{S}(\mathbf{H}))$ : $\prod_{k=1}^{M} \mathcal{C}_{k} \rightarrow \mathbb{R}_{+}$, and the beamforming functions $\mathbf{v}_{k}(\mathcal{S}(\mathbf{H}))$ : $\prod_{k=1}^{M} \mathcal{C}_{k} \rightarrow \mathfrak{U}_{M}$, where $\mathfrak{U}_{M}$ is the unit hypersphere in $\mathbb{R}^{M}$.

An exact solution to this problem is intractable. Our approach in solving this problem is a suboptimal one where we fix the outage regions of the users (with volumes $q_{k}$ ) and design the system such that outage is prevented in all other regions. Define the outage region $\Omega_{k} \subset \prod_{k} \mathcal{C}_{k}$ for user $k$ such that $\operatorname{prob}\left[\mathcal{S}(\mathbf{H}) \in \Omega_{k}\right]=q_{k}$ and let $I_{k}$ be the no-outage flag for user $k: I_{k}=\mathcal{I}\left(\mathcal{S}(\mathbf{H}) \in \Omega_{k}^{c}\right)$, where $\mathcal{I}(\cdot)$ is 1 if its logical argument is true and 0 otherwise.

Let us fix the codebook sizes $N_{k}$ for now. In order to prevent outage when $I_{k}=1$, we need to design the codebooks, the power control function, and the beamforming functions such that the target SINR's are guaranteed for the worst case:

$$
\begin{aligned}
& \min _{\substack{\mathcal{C}_{k}, P_{k}(\mathcal{S}(\mathbf{H})), \mathbf{v}_{k}(\mathcal{S}(\mathbf{H}))}} \mathbb{E}_{\mathbf{H}}\left[\sum_{k=1}^{M} P_{k}(\mathcal{S}(\mathbf{H}))\right] \\
& \text { s.t. } \quad \inf _{\mathbf{w} \in S_{k}\left(\mathbf{h}_{k}\right)} \frac{P_{k}(\mathcal{S}(\mathbf{H}))\left|\mathbf{w}^{T} \mathbf{v}_{k}(\mathcal{S}(\mathbf{H}))\right|^{2}}{\sum_{l \neq k} P_{l}(\mathcal{S}(\mathbf{H}))\left|\mathbf{w}^{T} \mathbf{v}_{l}(\mathcal{S}(\mathbf{H}))\right|^{2}+1}>\gamma_{k} I_{k},
\end{aligned}
$$

$$
\text { for all } \mathbf{H} \in \mathbb{R}^{M^{2}} \text { and } k=1,2, \cdots, M \text {. }
$$

Note that this formulation returns $P_{k}(\mathcal{S}(\mathbf{H}))=0$ if user $k$ is in outage, i.e. $I_{k}=0$.

The robust formulation in (13), although still intractable, reveals two main sources of outage:

- Magnitude outage: If the quantization region $S_{k}\left(\mathbf{h}_{k}\right)$ includes the zero vector $\mathbf{w}=0$, the constraint will not be feasible for user $k$ and the user will be in outage.

- Direction outage: Assume that there exists a vector $\mathbf{w}_{i} \in$ $S_{i}\left(\mathbf{h}_{i}\right)$ such that $\mathbf{w}_{j}=c \mathbf{w}_{i} \in S_{j}\left(\mathbf{h}_{j}\right)$ for some arbitrary constant $c$ and distinct users $i \neq j$. It is easy to verify that the SINR constraints $P_{i}\left|\mathbf{w}_{i}^{T} \mathbf{v}_{i}\right|^{2} / P_{j}\left|\mathbf{w}_{i}^{T} \mathbf{v}_{j}\right|^{2}>\gamma_{i}$ and $P_{j}\left|\mathbf{w}_{j}^{T} \mathbf{v}_{j}\right|^{2} / P_{i}\left|\mathbf{w}_{j}^{T} \mathbf{v}_{i}\right|^{2}>\gamma_{j}$ cannot coexist if $\gamma_{i}, \gamma_{j}>1$. Therefore, the users will also be in outage if their quantization regions are in near alignment with each other.

To present a tractable solution to the problem in (13), we resort to the product codebook structures as described in the next section.

\section{Channel Magnitude and Direction QUANTIZATION}

This section describes the product codebook structure and explicitly specifies the outage regions. For a given target outage probability $q$, the magnitude and direction outage regions are defined in a way that $q=\dot{q}+\ddot{q}$, where $\dot{q}$ and $\ddot{q}$ are respectively referred to as magnitude and direction outage probabilities.

\section{A. Channel Magnitude Quantization}

For each user $1 \leq k \leq M$, we use a magnitude quantization codebooks $\mathbb{Y}_{k}=\left\{y_{k}^{(1)}, y_{k}^{(2)}, \cdots, y_{k}^{\left(\dot{N}_{k}\right)}\right\}$ for quantizing the channel magnitude variable $Y_{k} \stackrel{\text { def }}{=}\left\|\mathbf{h}_{k}\right\|^{2}$, where $y_{k}^{(n)}$ are the magnitude quantization levels and $\dot{N}_{k}$ is the magnitude codebook size.

Given a magnitude outage probability $\dot{q}$, we fix the interval $\left[0, y_{k}^{(1)}\right)$ as the magnitude outage region. The first quantization level is therefore fixed as

$$
y_{k}^{(1)}=F_{k}^{-1}\left(\dot{q}_{k}\right),
$$

where $F_{k}^{-1}(\cdot)$ is the inverse cdf of $Y_{k} \stackrel{\text { def }}{=}\left\|\mathbf{h}_{k}\right\|^{2}$. The following definitions are used in describing the product codebook structure.

Define $\dot{\mathcal{C}}_{k}$ as the set of all quantization intervals except the outage interval:

$$
\dot{\mathcal{C}}_{k}=\left\{J_{k}^{(1)}, J_{k}^{(2)}, \cdots, J_{k}^{\left(\dot{N}_{k}\right)}\right\},
$$

where $J_{k}^{(n)}=\left[\sqrt{y_{k}^{(n)}}, \sqrt{y_{k}^{(n+1)}}\right)$ and $y_{k}^{\left(\dot{N}_{k}+1\right)} \stackrel{\text { def }}{=} \infty$. Note that the definition uses the square root of the levels as the quantization levels $y_{k}^{(n)}$ are defined for quantizing $\left\|\mathbf{h}_{k}\right\|^{2}$.

Finally, for $Y_{k} \geq y_{k}^{(1)}$, define the quantized magnitude $\tilde{Y}_{k}$ as the quantization level in $\mathbb{Y}_{k}$ that is in the immediate left of $Y_{k}$. The quantized variable $\tilde{Y}_{k}$ is exactly the same as the parameter $r_{k}$ in the discussion of the robust power control problem in Section III.

The ultimate goal of this paper is to optimize the magnitude and direction quantization codebooks such that the average sum power is minimized. For this purpose, as it will be clarified in Section VI, we use the expectation of the upper bound in Theorem 2 as the average sum-power upper bound. According to (11), the average terms $\mathbb{E}\left[e_{k}\right]$ and $\mathbb{E}\left[f_{k}\right]$ include the term $\mathbb{E}\left[1 / r_{k}\right]$. We are therefore interested in a magnitude quantization codebook $\mathbb{Y}$ that minimizes $\mathbb{E}\left[1 / \tilde{Y}_{k}\right]$.

It is shown in [6] that, in the asymptotic regime where $\dot{N}_{k} \rightarrow \infty$, the optimal magnitude levels in $\mathbb{Y}$ form a geometric sequence, i.e. the asymptotically optimal levels are uniformly spaced in dB scale. As it is shown in [6], this optimality is not affected by the channel magnitude distribution as long as some regularity conditions are satisfied. Moreover, assuming $\dot{N}_{k} \gg$ 1, we have the following for such an optimal codebook $\mathbb{Y}$ :

$$
\mathbb{E}\left[\frac{1}{\tilde{Y}_{k}}\right]<\rho_{\text {CSI }}\left(1+\dot{N}_{k}^{-\zeta_{k}\left(\dot{N}_{k}\right)}\right),
$$

where $\rho_{\text {CSI }}$ is defined in (3) and the function $\zeta_{k}\left(\dot{N}_{k}\right)$, as defined in [6], is a function that depends on the probability distribution function of $Y_{k}$ and has the property

$$
\lim _{\dot{N}_{k} \rightarrow \infty} \zeta_{k}\left(\dot{N}_{k}\right)=1 .
$$

\section{B. Channel Direction Quantization}

For channel direction quantization, we use $M$-dimensional Grassmannian codebooks $\mathbb{U}_{k}$ for users $1 \leq k \leq M$ :

$$
\mathbb{U}_{k}=\left\{\mathbf{u}_{k}^{(1)}, \mathbf{u}_{k}^{(2)}, \cdots, \mathbf{u}_{k}^{\left(\ddot{N}_{k}\right)}\right\},
$$

where $\mathbf{u}_{k}^{(n)}$ vectors are $M$-dimensional unit-norm Grassmannian codewords and $\ddot{N}_{k}$ is the direction quantization codebook 
size. The direction quantization regions are formed by mapping each channel vector $\mathbf{h}_{k}$ to a vector $\tilde{\mathbf{u}}_{k}\left(\mathbf{h}_{k}\right) \in \mathbb{U}_{k}$ that has the smallest angle with $\mathbf{h}_{k}$ :

$$
\tilde{\mathbf{u}}_{k}\left(\mathbf{h}_{k}\right)=\arg \min _{\mathbf{u} \in \mathbb{U}_{k}} \angle\left(\mathbf{h}_{k}, \mathbf{u}\right) .
$$

We refer to $\tilde{\mathbf{u}}_{k}\left(\mathbf{h}_{k}\right)$ as the quantized direction for the channel realization $\mathbf{h}_{k}$. The corresponding quantization regions, according to the Gilbert-Varshamov bound argument [10], can be covered by the following spherical caps:

$$
\ddot{\mathcal{C}}_{k}=\left\{B_{k}^{(1)}, B_{k}^{(2)}, \cdots, B_{k}^{\left(\ddot{N}_{k}\right)}\right\}
$$

where $B_{k}^{(n)}=\left\{\mathbf{w} \mid\|\mathbf{w}\|=1, \angle\left(\mathbf{w}, \mathbf{u}_{k}^{(n)}\right)<\arcsin \delta_{k}\right\}$, and $\delta_{k}$ is the minimum chordal distance of $\mathbb{U}_{k}$. This covering (enlargement) of the regions increases the required transmission power, which is in the direction of our analysis of deriving sum-power upper bounds.

In order to use the results in Section III, we define $\phi_{k}$, which are referred to as uncertainty angles in Section III, as follows:

$$
\phi_{k} \stackrel{\text { def }}{=} \arcsin \delta_{k} .
$$

We will need the following bound in optimizing the product codebook structure in Section VI.

Lemma 1: For a $M$-dimensional real Grassmannian codebook of size $\ddot{N}$ and minimum chordal distance $\delta$, we have the following for large enough $\ddot{N}$ :

$$
\delta<4 \lambda_{M} \ddot{N}^{-\frac{1}{M-1}},
$$

where $\lambda_{M}=\left(\frac{\sqrt{\pi} \Gamma((M+1) / 2)}{\Gamma(M / 2)}\right)^{\frac{1}{M-1}}$ and $\Gamma(\cdot)$ is the gamma function.

Proof: Proof is based on the Hamming bound as in [10].

By combining (20) and Lemma 1, we have the following for small $\phi_{k}$ and large enough $\ddot{N}_{k}$ :

$$
\phi_{k}<4 \lambda_{M} \ddot{N}_{k}^{-\frac{1}{M-1}} \text {. }
$$

To address the direction outage, we assume that the beamforming vectors are the zero-forcing vectors for the quantized directions $\tilde{\mathbf{u}}_{k}=\tilde{\mathbf{u}}\left(\mathbf{h}_{k}\right)$. We say that user $k$ is in direction outage if $0 \leq \theta_{k} \leq \theta_{k}^{\circ}$, where $\theta_{k}=\angle\left(\tilde{\mathbf{u}}_{k}, \tilde{\mathbf{U}}_{-k}\right)$ as defined in Section III, and $\theta_{k}^{\circ} \ll 1$ is the minimum acceptable angle between $\tilde{\mathbf{u}}_{k}$ and $\tilde{\mathbf{U}}_{-k}$. This implicitly defines the direction outage regions. Moreover, by assuming that $\theta_{k}$ is approximately uniform ${ }^{1}$ in $[0, \pi / 2]$, the direction outage probability is given by

$$
\ddot{q}_{k} \approx \frac{2}{\pi} \theta_{k}^{\circ}
$$

For all other no-outage cases, i.e. $\theta_{k} \geq \theta_{k}^{\circ}$, all the SINR constraints should be feasible. By using the sufficient feasibility condition in (9) and noting that user $k$ is not aware of

\footnotetext{
${ }^{1}$ This holds if the codebooks $\mathbb{U}_{k}$ undergo sufficient random rotations.
}

$\theta_{k}$ and the codebook structures are fixed, the parameters $\phi_{k}$ should be set for the worst case as follows:

$$
\phi_{k}<\frac{\theta_{k}^{\circ}}{1+\sqrt{(M-1) \gamma_{k}}} .
$$

This justifies the assumption of $\phi_{k} \ll 1$ in Theorem 2 . Now, by considering the potential range of $\phi_{k}$ in (22) and using (24), we have the following sufficient condition on $\ddot{N}_{k}$ in order to guarantee the SINR targets for the no-outage cases:

$$
\ddot{N}_{k} \geq\left(\frac{4 \lambda_{M}}{\theta_{k}^{\circ}}\left(1+\sqrt{(M-1) \gamma_{k}}\right)\right)^{M-1} .
$$

\section{PRoduct CODEBOOK OPTIMIZATION AND ASYMPTOTIC BIT-SHARING RULES}

By recalling the definitions in Sections IV and $\mathrm{V}$, the product codebooks for users $1 \leq k \leq M$ are formed as follows:

$$
\mathcal{C}_{k}=\left(\dot{\mathcal{C}}_{k} \times \ddot{\mathcal{C}}_{k}\right) \cup O_{k},
$$

where $\dot{\mathcal{C}}_{k}$ and $\ddot{\mathcal{C}}_{k}$ are the magnitude and direction codebooks in (15) and (19) and $O_{k}=\left\{\mathbf{h} \mid\|\mathbf{h}\|^{2}<y_{k}^{(1)}\right\}$. Moreover, the no-outage flag for user $k$ is

$$
I_{k}=\mathcal{I}\left(\theta_{k}>\theta_{k}^{\circ} \wedge\|\mathbf{h}\|^{2}>y_{k}^{(1)}\right),
$$

where $\mathcal{I}(\cdot)$ is the logic true function. Finally, the outage probability of user $k$ is given by

$$
q_{k}=\dot{q}_{k}+\ddot{q}_{k} \approx F_{k}\left(y_{k}^{(1)}\right)+\frac{2}{\pi} \theta_{k}^{\circ} .
$$

Now, for every realization of the random channels $\mathbf{H}=$ $\left[\mathbf{h}_{1}, \mathbf{h}_{2}, \cdots, \mathbf{h}_{M}\right]$, we can bound the instantaneous solution of (13) with the upper bound solution (10) by replacing the variables $\gamma_{k}, r_{k}, \zeta_{k}$ with the random variables $\gamma_{k} I_{k}, \tilde{Y}_{k}$, and $\cot \theta_{k}$, where the random variables $\tilde{Y}_{k}$ and $\theta_{k}$ are defined in Sections V.A and V.B.

If we ignore the last term in (10) and take the expectation of $P_{u b}$ with respect to $I_{k}, \tilde{Y}_{k}, \theta_{k}$, and use (16) and (22), we achieve the following upper bound:

$$
\begin{aligned}
\mathbb{E}\left[P_{u b}\right] & <\frac{2 \rho_{C S I}}{\pi} \sum_{k=1}^{M} \frac{\gamma_{k}}{\theta_{k}^{\circ}}\left(1+\dot{N}_{k}^{-\zeta_{k}\left(\dot{N}_{k}\right)}+\frac{4 \lambda_{M}}{\theta_{k}^{\circ}} \ddot{N}_{k}^{-\frac{1}{M-1}}\right) \\
& \approx \frac{2 \rho_{C S I}}{\pi} \sum_{k=1}^{M} \frac{\gamma_{k}}{\theta_{k}^{\circ}}\left(1+\dot{N}_{k}^{-1}+\frac{4 \lambda_{M}}{\theta_{k}^{\circ}} \ddot{N}_{k}^{-\frac{1}{M-1}}\right),
\end{aligned}
$$

where we have assumed $\dot{N}_{k}, \ddot{N}_{k} \gg 1$ and used (17) in deriving (30).

Assume a total feedback rate constraint $B$ such that $\prod_{k=1}^{M} \ddot{N}_{k} \dot{N}_{k} \leq 2^{B}$. We want to minimize the average sumpower upper bound in (30) with respect to this rate constraint, the target outage constraints (28), and the sufficient (no-outage) constraints (25), in the asymptotic regime of $B, \dot{B}_{k}, \ddot{B}_{k} \gg 1$, where $\dot{B}_{k} \stackrel{\text { def }}{=} \log \dot{N}_{k}$ and $\ddot{B}_{k} \stackrel{\text { def }}{=} \log \ddot{N}_{k}$.

In order to satisfy the outage target constraints, we fix $y_{k}^{(1)}$ and $\theta_{k}^{\circ}$ such that $F_{k}\left(y_{k}^{(1)}\right)=\frac{2}{\pi} \theta_{k}^{\circ}=\frac{q_{k}}{2}$, i.e. we make the simplifying assumption that the magnitude and direction outage are equally likely. It can be shown that any other linear 
division of $q_{k}$ between $\dot{q}_{k}$ and $\ddot{q}_{k}$ only affects the optimal $\ddot{B}_{k}$ and $\dot{B}_{k}$ by a fixed bounded number of bits, i.e. the variable $\eta$ in equations (31) and (32).

By minimizing (30) subject to $\sum_{k} \dot{B}_{k}+\ddot{B}_{k}=B$ we have the following result:

Theorem 3: Define

$$
\begin{aligned}
& \dot{B}_{\mathrm{ave}}=\frac{1}{M^{2}} B-\frac{M-1}{M} \log \frac{1}{\bar{q}}-\eta \\
& \ddot{B}_{\mathrm{ave}}=\frac{M-1}{M^{2}} B+\frac{M-1}{M} \log \frac{1}{\bar{q}}+\eta,
\end{aligned}
$$

where $\eta=\frac{M-1}{M} \log \frac{16 \lambda_{M}}{\pi(M-1)}$ and $\bar{q}=\sqrt[M]{\prod_{k} q_{k}}$. The optimal values of $\dot{B}_{k}$ and $\ddot{B}_{k}$ are given by

$$
\begin{aligned}
& \dot{B}_{k}=\dot{B}_{\text {ave }}+\log \frac{\gamma_{k}}{\bar{\gamma}}+\log \frac{\bar{q}}{q_{k}} \\
& \ddot{B}_{k}=\ddot{B}_{\text {ave }}+(M-1) \log \frac{\gamma_{k}}{\bar{\gamma}}+2(M-1) \log \frac{\bar{q}}{q_{k}},
\end{aligned}
$$

where $\bar{\gamma}=\sqrt[M]{\prod_{k} \gamma_{k}}$.

Corollary 1: For each user $k$, the optimal number of magnitude and direction quantization bits are related as follows:

$$
\ddot{B}_{k}=(M-1) \dot{B}_{k}+(M-1) \log \frac{1}{q_{k}}+M \eta .
$$

In the asymptotic regime of $B \rightarrow \infty$, the number of direction quantization bits $\ddot{B}_{k}$ is therefore $M-1$ times the number of magnitude quantization bits $\dot{B}_{k}$. Moreover, the total number of quantization bits for user $k$ is given by

$$
B_{k}=\dot{B}_{k}+\ddot{B}_{k}=\frac{1}{M} B+M \log \frac{\gamma_{k}}{\bar{\gamma}}+(2 M-1) \log \frac{\bar{q}}{q_{k}}
$$

which shows that the $k$ th user's share of the total feedback rate is controlled by $\log \gamma_{k}$ and $\log 1 / q_{k}$.

By forcing the optimal values of $\ddot{N}_{k}=2^{\ddot{B}_{k}}$ to satisfy the sufficient (no-outage) constraint in (25), we achieve the following minimum required total feedback rate $B$ :

Theorem 4: Assume $\gamma_{k}>1$. For the target SINR's $\gamma_{k}$ to be feasible with outage probabilities $q_{k}$, the following total feedback rate $B$ is sufficient:

$$
B>\frac{1}{2} M^{2} \log \bar{\gamma}+\left(M^{2}-M\right) \log \frac{1}{\bar{q}}+M^{2} \log \Delta+b,
$$

where $\bar{\gamma}$ and $\bar{q}$ are the geometric means of $\gamma_{k}$ 's and $q_{k}$ 's respectively,

$$
\Delta=\max _{1 \leq k \leq M} \frac{q_{k} / \sqrt{\gamma_{k}}}{\bar{q} / \sqrt{\bar{\gamma}}}
$$

and $b=\frac{1}{2} M^{2}+\frac{3}{2} M^{2} \log M+M^{2} \eta$ with $\eta$ is defined in Theorem 3 .

Theorem 4 shows that the system feedback link capacity should increase logarithmically with $\bar{\gamma}$ and $\bar{q}$. Comparing with the users that have similar target parameters $\gamma_{k}=\bar{\gamma}$ and $q_{k}=\bar{q}$, the users with different target parameters impose an additional requirement, $M^{2} \log \Delta$, on the total feedback rate. The more the target parameters deviate from the average parameters $\bar{q}$ and $\bar{\gamma}$, the higher is this requirement.
Finally, by substituting the optimal values of $\ddot{B}_{k}$ and $\dot{B}_{k}$ in (30), we have the following result which shows the scaling of the average sum power with $B$ :

Theorem 5: For a system with $M$ antennas at the base station and a total feedback rate $B$, we have

$$
\mathbb{E}\left[P_{u b}\right]<P_{\text {CSI }}\left(1+\frac{\kappa}{\bar{q}} \cdot 2^{-\frac{B}{M^{2}}}\right),
$$

where $P_{\text {CSI }}$ is defined in (2), $\bar{q}=\sqrt[M]{\prod_{k} q_{k}}$, and

$$
\begin{gathered}
\kappa=\frac{16 M}{\pi(M-1)}\left(\frac{\pi^{3 / 2}(M-1) \Gamma((M+1) / 2)}{16 \Gamma(M / 2)}\right)^{1 / M} . \\
\text { VII. ConCLUSIONS }
\end{gathered}
$$

This paper studies the channel quantization codebook optimization for multiuser spatial multiplexing system with $M$ antennas at the base station and a total feedback rate $B$. It is shown that in the asymptotic regime of $B \rightarrow \infty$, the optimal number of direction quantization bits is $M-1$ times the number of magnitude quantization bits. As a general rule, a user with a higher QoS (lower target outage probability) and higher target rate (higher target SINR) needs a higher channel quantization resolution. The paper also shows that the total required feedback rate increases logarithmically with the geometric mean of the target SINR's $\bar{\gamma}$ and the geometric mean of the inverse outage probabilities $1 / \bar{q}$. The more the users' target parameters deviate from the mean parameters $\bar{\gamma}$ and $\bar{q}$, the more is the required feedback. The paper also derives the scaling of the system performance with the feedback rate.

\section{REFERENCES}

[1] D. J. Love, R. W. Heath, V. K. N. Lau, D. Gesbert, B. D. Rao, and M Andrews, "An overview of limited feedback in wireless communication systems," IEEE J. Select. Areas Commun., vol. 26, no. 8, pp. 1341-1365, Oct. 2008.

[2] N. Jindal, "MIMO broadcast channels with finite rate feedback," IEEE Trans. Inform. Theory, vol. 52, no. 11, pp. 5045-5059, Nov. 2006.

[3] T. Yoo, N. Jindal, and A. Goldsmith, "Multi-antenna downlink channels with limited feedback and user selection," IEEE J. Select. Areas Commun., vol. 25, no. 7, pp. 1478-1491, Sep. 2007.

[4] K. Huang, R. W. Heath, and J. G. Andrews, "Space division multiple access with a sum feedback rate constraint," IEEE Trans. Sig. Proc., vol. 55, no. 7, pp. 3879-3891, Jul. 2007.

[5] G. Caire, N. Jindal, M. Kobayashi, and N. Ravindran, "Multiuser MIMO achievable rates with downlink training and channel state feedback," IEEE Trans. Inform. Theory, to appear.

[6] B. Khoshnevis and W. Yu, "High resolution quantization codebook design for multiple-antenna fading channels," to appear in Proc. 25th Biennial Symposium on Communications (QSBC), Kingston, Canada, May 2010.

[7] B. Khoshnevis and W. Yu, "Joint power control and beamforming codebook design for MISO channels with limited feedback," Global Communications Conference (Globecom), Honolulu, HI, Nov. 30-Dec. 4, 2009.

[8] B. Khoshnevis and W. Yu, "Structure of channel quantization codebook for multiuser spatial multiplexing systems," to appear in Proc. IEEE International Conference on Communications (ICC), Cape Town, South Africa, May 2010.

[9] B. Khoshnevis and Wei Yu, "Quantization codebook design for multipleantenna channels-part II: multiser system," preprint.

[10] D. J. Love and R. W. Heath, "Grassmannian beamforming for multipleinput multiple-output wireless systems," IEEE Trans. Inform. Theory, vol. 49, no. 10, pp. 2735-2747, Oct. 2003. 